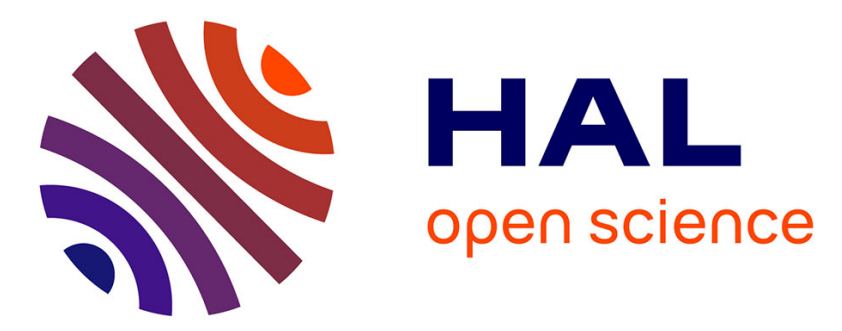

\title{
Les instabilités magnétiques sur un échantillon court de $\mathrm{Nb}-\mathrm{Ti}$
}

\author{
I. Hlasnik, D. Rappanello
}

\section{To cite this version:}

I. Hlasnik, D. Rappanello. Les instabilités magnétiques sur un échantillon court de Nb-Ti. Revue de Physique Appliquée, 1971, 6 (4), pp.445-452. 10.1051/rphysap:0197100604044500 jpa-00243569

\section{HAL Id: jpa-00243569 https://hal.science/jpa-00243569}

Submitted on 1 Jan 1971

HAL is a multi-disciplinary open access archive for the deposit and dissemination of scientific research documents, whether they are published or not. The documents may come from teaching and research institutions in France or abroad, or from public or private research centers.
L'archive ouverte pluridisciplinaire HAL, est destinée au dépôt et à la diffusion de documents scientifiques de niveau recherche, publiés ou non, émanant des établissements d'enseignement et de recherche français ou étrangers, des laboratoires publics ou privés. 


\title{
LES INSTABILITÉS MAGNÉTIQUES SUR UN ÉCHANTILLON COURT DE Nb-Ti
}

\author{
par I. HLASNIK * et D. RAPPANELLO \\ Département Saturne/G. A. T.S/C.E. A. de Saclay \\ (Reçu le 29 avril 1971, révisé le 8 juillet 1971)
}

\begin{abstract}
Résumé. - On montre ici, à partir d'expériences réalisées sur échantillons courts (Nb-Ti) traversés par un courant de transport, l'influence déterminante des instabilités magnétiques sur les caractéristiques nominales du supraconducteur. L'analyse mathématique des essais confirme le modèle adiabatique théorique adopté : sur une configuration géométrique simple, on détermine un critère de stabilité vérifié par l'expérience faite sur des matériaux stabilisés au $\mathrm{Pb}$ et $\mathrm{Au}$. On conclut par une première approche du phénomène de training que l'on relie à des courants diamagnétiques dans le supraconducteur.
\end{abstract}

Abstract. - One shows, from experiments on short samples (Nb-Ti) with transport current, the determinant influence of magnetic instabilities on the nominal characteristics of the superconducting materials. The mathematical analysis of these experiences agrees with the theoretical adiabatic used model : from a simple geometrical shape a stability criterion is determined which is verified by tests done on material stabilized with $\mathrm{Pb}$ and $\mathrm{Au}$. One concludes giving a first approach of the training phenomenon in connection with diamagnetic currents in the superconducting material.

I. Introduction. - Les instabilités magnétiques peuvent apparaître dans un matériau supraconducteur de $2^{e}$ espèce lorsqu'il est placé dans un champ magnétique variable, comme conséquence des pertes qui apparaissent alors dans le supraconducteur. Ce phénomène appelé saut de flux apparaissant pour des valeurs déterminées d'induction magnétique a été observé par Kim et ses collaborateurs [1] sur des cylindres creux. L'analyse théorique a été réalisée par plusieurs auteurs, Hancox [2], Wipf [3]. Swartz et Bean [4] pour le cas adiabatique. Ces auteurs ont déterminé les conditions d'existence et d'apparition de ces sauts de flux sur un semi-espace et sur une plaque semi-infinie. Les études de Bean et Wipf sont effectuées en absence de courant de transport dans le matériau supraconducteur. Hancox [5] sur le même modèle trouve un résultat comparable à un facteur 1,11 près au résultat de Swartz et Bean. Pareillement, Hancox [6] a étendu le critère d'apparition de saut de flux au cas général du bobinage, l'assimilant à une plaque unique et dont la pénétration de champ au moment du saut de flux ne se fait pas que d'un côté de la plaque, mais à partir de la pénétration totale du champ dans le matériau. Smith [7] et ses collaborateurs

$\left.{ }^{*}\right)$ Collaborateur étranger de l'Institut d'Electrotechnique de l'Académie Slovaque des Sciences de Bratislava (Tchécoslovaquie). ont élargi le calcul de Hancox aussi dans le cas où le champ polarisant n'a pas complètement pénétré le supraconducteur.

Dans notre travail, nous voulons montrer que les conditions d'apparition d'un saut de flux sous l'influence d'un courant de transport et son évolution sur un échantillon court d'un fil rond (refroidi et non refroidi dans un champ magnétique extérieur et pour différentes valeurs de température du réfrigérant), sont un peu différentes de celles mises en évidence dans les cas cités plus haut. A partir de nos résultats expérimentaux, nous présentons deux modèles théoriques, l'un en coordonnées cylindriques, l'autre en considérant une plaque infinie, et des calculs numériques corroborant les expériences. Ensuite, en adoptant le modèle de la plaque infinie qui permet des calculs plus simples, on étend la discussion au cas où le fil est soumis après la mise en froid, à une variation $\Delta H_{\mathrm{ex}}$ du champ extérieur. L'effet de ce $\Delta H_{\mathrm{ex}}$ revient en fait à introduire le courant de transport avec déjà au sein du supraconducteur des courants diamagnétiques. En guise de conclusion, on compare, tout d'abord, les deux modèles proposés entre eux. On termine ensuite par la comparaison des résultats numériques obtenus à partir du modèle élaboré sur la plaque avec, d'une part, des expériences réalisées sur le matériau stabilisé au $\mathrm{Pb}$ et $\mathrm{Au}$, et d'autre part, avec les expériences réalisées à partir du même type de matériau supraconducteur dont la section a été diminuée. 
II. Les expériences. - Le supraconducteur utilisé est du type $\mathrm{Nb} 40 \%$ Ti de $220 \mu$ de section non enrobé de cuivre. L'échantillon est monté sur un dispositif constitué par une cellule à température variable contenant du gaz hélium, de telle façon que le courant qui le traverse soit perpendiculaire au champ de polarisation appliqué. Le bloc thermométrique en cuivre dans lequel est encastré l'échantillon supraconducteur possède un système permettant de faire varier la température. L'échantillon est thermiquement en contact avec le bloc de chauffage de telle façon que la mesure de température effectuée simultanément sur le bloc de chauffage et sur l'échantillon par une résistance AllenBradley collée au vernis GE 7021 représente effectivement la température du fil supraconducteur dans le régime stationnaire. Dans le régime transitoire tel qu'un saut de flux, par contre, les échanges thermiques entre, d'une part, le fil et le bloc de cuivre, la résistance Allen-Bradley et l'hélium gazeux d'autre part, sont négligeables.

La méthode de mesure est classique : à chaque valeur d'induction et de température affichée, on détermine l'intensité maximum du courant susceptible de passer dans le supraconducteur. Nous réalisons avec le même matériau, mais non le même échantillon, deux types d'expériences : tout d'abord sur échantillon A, nous ne nous sommes pas préoccupés de l'histoire magnétique et thermique du matériau, et dans une autre mesure, échantillon $\mathrm{B}$, le matériau est systématiquement refroidi dans le champ polarisant avant introduction du courant de transport.

II. 1 EChANTILlon A NON REFroId DANS $H$. - Sur la figure 1, échantillon $A$, pour le cas non refroidi dans le champ, on porte, en fonction de la température du milieu réfrigérant et pour chaque valeur de l'induction, les intensités des courants entraînant l'apparition de l'état normal. Ces valeurs sont celles qui sont maximales et les plus reproductibles après le training.

- En traits pleins, on trace les points de mesure obtenus.

- En traits pointillés, les points obtenus lorsque le matériau est stabilisé au $\mathrm{Pb}$.

Il apparaît alors que la loi $J_{\mathrm{c}}(T)$ est une droite, sauf au voisinage immédiat de $T_{\mathrm{c}}$; résultats en accord avec les travaux de Hampshire [8] et Yamafuji [9]. Par ailleurs, il existe un training très marqué dans certaines zones de température et de champ ( $T$ et $H$ faibles) auxquelles se superpose une dégradation des courants mesurés par rapport aux courants critiques nominaux. Cette dégradation est elle aussi décroissante en fonction du champ et de la température. Ceci indique clairement que le supraconducteur en échantillon court placé dans les conditions expérimentales décrites est le siège d'instabilités thermiques ou magnétiques entraînant la transition brutale et intempestive de tout l'échantillon.

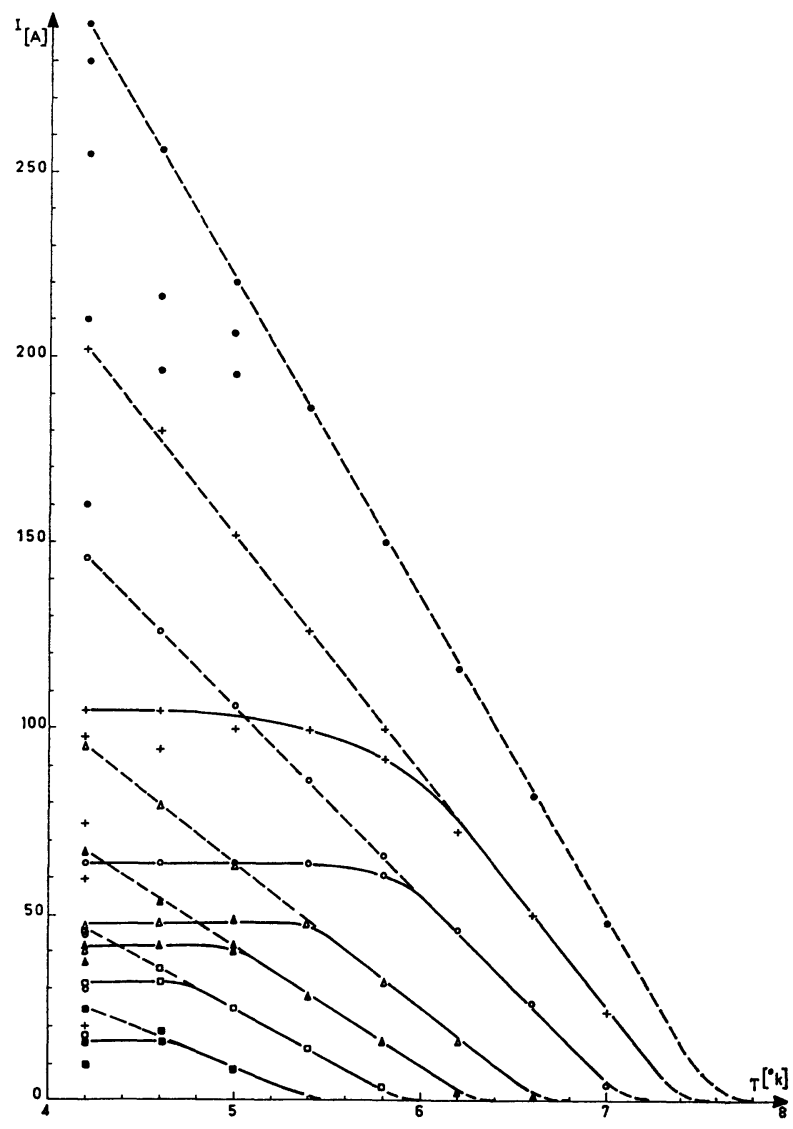

FIG. 1. $-I=f[T][B]$. Echantillon A. (Nb $40 \%$ Ti) $\varnothing 220 \mu$. Non refroidi dans $H$.

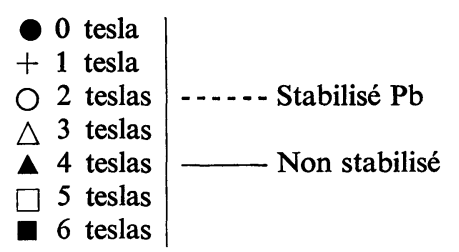

II.2. Echantillon B Refroidi Dans $H$. - Pour bien définir les conditions initiales, nous refroidissons de manière systématique l'échantillon court $B$ dans le champ appliqué avant toute introduction de courant de transport. Dans ce cas, le courant de transition apparaît de façon reproductible à une valeur bien déterminée et ne présentant pas de dispersion, figure 2. Le training, dans ce mode opératoire là, ne se manifeste pas. On peut noter aussi que les valeurs des courants de blocage obtenues dans cette mesure sont identiques à celles obtenues après training pour l'échantillon $\mathrm{B}$, dans le cas effectivement mesuré et porté sur la figure 2, où il n'est pas refroidi dans le champ.

Par ces deux modes opératoires, on a mis en évidence l'influence déterminante des courants diamagnétiques sur les courants de blocage créés parjla variation du champ polarisant après le refroidissement du matériau. On se propose d'associer les courants de blocage ainsi déterminés aux instabilités magnétiques; celles-ci peuvent être reliées à la pénétration du champ magnétique propre dû au courant de transport. La 


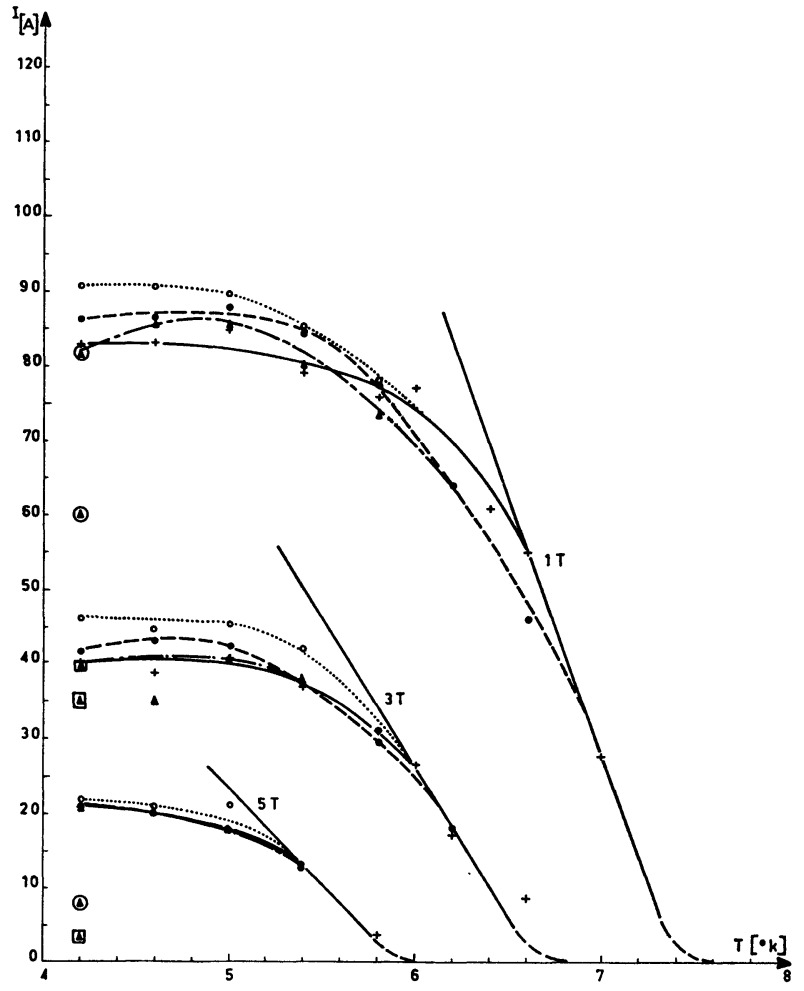

FIG. 2. $-I_{\mathrm{b}}=f[T B]$. Echantillon B. $(\mathrm{Nb} 40 \% \mathrm{Ti})$. $\varnothing 220 \mu$.

\begin{tabular}{|c|c|c|c|c|}
\hline \multirow[t]{2}{*}{ Expérience } & \multicolumn{2}{|c|}{$+\longrightarrow$} & \multicolumn{2}{|c|}{$\begin{array}{l}\text { Refroidi dans } H \text {. } \\
\text { Non refroidi dans } H \text {. }\end{array}$} \\
\hline & (4) & 1 & tesla & $C_{1 \mathrm{~T}}=4,5 \mathrm{~mJ} / \mathrm{cm}^{3}{ }^{\circ} \mathrm{K}$ \\
\hline $4,2{ }^{\circ} \mathrm{k}$ & $\frac{\Delta}{\Delta}$ & $\begin{array}{l}3 \\
5\end{array}$ & $\begin{array}{l}\text { teslas, avec } \\
\text { teslas }\end{array}$ & $\begin{array}{l}C_{3 \mathrm{~T}}=1,5 \mathrm{~mJ} / \mathrm{cm}^{3}{ }^{\circ} \mathrm{K} \\
C_{5 \mathrm{~T}}=0,5 \mathrm{~mJ} / \mathrm{cm}^{3}{ }^{\circ} \mathrm{K}\end{array}$ \\
\hline Théorie & & 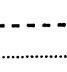 & Refroidi & $\begin{array}{l}\text { dèle fil rond. } \\
\text { dèle plaque. }\end{array}$ \\
\hline
\end{tabular}

différence essentielle entre nos essais et les différents travaux [2], [3], [4], [7] relatifs aux sauts de flux réside dans la mise en évidence de ces mêmes instabilités à partir de l'étude directe des courants de transport dans les échantillons courts. Le modèle théorique élaboré ici, dans le cas d'une géométrie cylindrique, est susceptible d'expliquer ces résultats.

III. Théorie. - Pour un fil rond, refroidi dans un champ magnétique extérieur $H_{\mathrm{ex}}$ la pénétration du courant se fera régulièrement de toute la surface vers l'intérieur (Fig. 3-1) ; elle se fera de façon plus compliquée (Fig. 3-2) lorsque le fil est refroidi avant l'affichage de $H_{\mathrm{ex}}$. Nous calculons ici (échantillon refroidi dans $H$ ) les courants instables $I_{\text {ins }}$ et les courants de blocage $I_{\mathrm{b}}$ pouvant être différents du courant critique $I_{c}$, dans le cas où les conditions adiabatiques sont vérifiées pour les variations brusques du courant et de sa répartition dans le matériau.

III.1 Fil ROND (REFROIDI DANS LE CHAMP). - Soit un fil rond de rayon extérieur $R$ refroidi dans le champ magnétique transversal $H_{\mathrm{ex}}$ tel que

$$
H_{\mathrm{ex}}+H_{0} \gg I_{\mathrm{b}} / 2 \pi R
$$

REVUE de PHYSIQUe APPLIQUée. - T. 6, No 4, DÉCEMBRe 1971

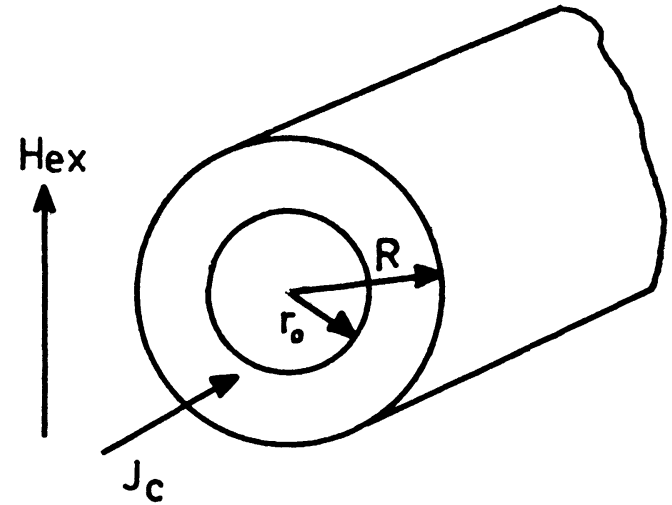

Fig. 3-1. $-I=\pi\left[R^{2}-r_{0}^{2}\right] J_{\mathrm{c}}$.

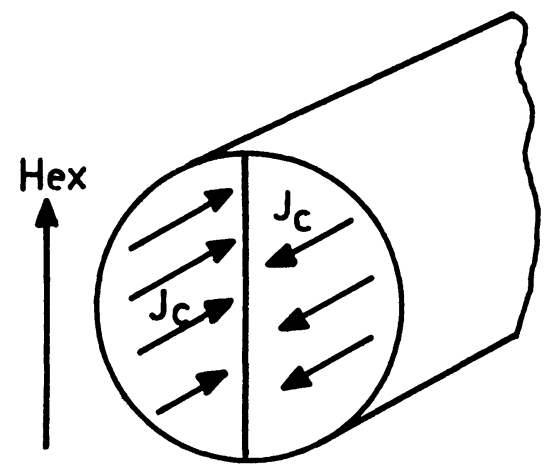

(a)

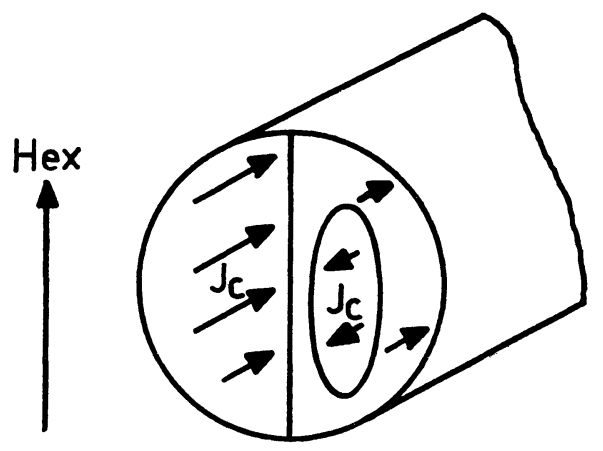

(b)

FIG. 3-2. - a) $I=0, b) I \neq 0$.

où $H_{0}$ est la constante de Kim. Nous pouvons supposer alors $J_{\mathrm{c}}$ pratiquement constant dans tout le volume du fil. Le courant va successivement pénétrer de la surface du fil jusqu'au rayon $r_{0}$ tel que

$$
I=\pi . J_{\mathrm{c}}\left[R^{2}-r_{0}^{2}\right] \text { ! }
$$

Le champ magnétique $H_{I}$ dû au courant $I$ dans la couche entre $r_{0}$ et $R$ (couche de pénétration) sera donné par l'expression suivante :

$$
H_{I}=\frac{I-\pi\left(R^{2}-r^{2}\right) J_{\mathrm{c}}}{2 \pi r}=\frac{J_{\mathrm{c}} r}{2}-\frac{I_{\mathrm{c}}-I}{2 \pi r} .
$$

Comme pour $r \ll r_{0}$ le champ électrique $E$ est nul, dans la couche de pénétration $E$ peut être exprimé comme :

$$
E=\int_{r_{0}}^{r} \mu_{0} \frac{\partial H_{I}}{\partial t} \mathrm{~d} r=\frac{\mu_{0}}{2 \pi} \frac{\partial I}{\partial t} \log \frac{r}{r_{0}}
$$


La densité volumique de puissance $p$ dissipée dans la couche de pénétration sera donnée par :

$$
p=E J_{\mathrm{c}}=\frac{\mu_{0} J_{\mathrm{c}}}{\mathrm{L}^{2} \pi} \frac{\partial I}{\partial t} \log \frac{r}{r_{0}} .
$$

La densité volumique des pertes $\Delta W$ dissipée pendant un changement de courant $\Delta I$ sera donnée par :

$$
\Delta W=\frac{\mu_{0} J_{\mathrm{c}}}{2 \pi} \Delta I \log \frac{r}{r_{0}} .
$$

En supposant que $\Delta I$ se produit dans un temps très court durant lequel les pertes apparues ne peuvent diffuser ni dans le volume du supraconducteur, ni dans le milieu environnant, à l'aide de (5), on détermine l'échauffement $\Delta T$ correspondant à la variation $\Delta I$ dans toute la couche de pénétration :

Soit

$$
\Delta T=\frac{\Delta W}{C}=\frac{\mu_{0} J_{\mathrm{c}}}{2 \pi C} \Delta I \log \frac{r}{r_{0}}
$$

où $C$ est la chaleur spécifique du supraconducteur par unité de volume. A cet échauffement $\Delta T$ correspond une diminution $\Delta J_{\mathrm{c} \Delta \mathrm{T}}$ de $J_{\mathrm{c}}$ :

$$
\Delta J_{\mathrm{c} \Delta T}=-\frac{\partial J_{\mathrm{c}}}{\partial T} \Delta T=-\frac{\partial J_{\mathrm{c}}}{\partial T} \frac{\mu_{0} J_{\mathrm{c}}}{2 \pi C} \Delta I \log \frac{r}{r_{0}}
$$

$\partial J_{\mathrm{c}} / \partial T$ étant constant dans tout le volume de la couche de pénétration, ce qui est vrai dans la plupart des cas, l'intégration de $\Delta J_{\mathrm{c} \Delta \mathrm{T}}$ dans toute la section de la couche de pénétration donne une diminution $\Delta I_{\Delta \mathrm{T}}$ du courant due à l'échauffement :

$$
\begin{aligned}
\Delta I_{\Delta T}=\int_{r_{0}}^{R} \Delta J_{\mathrm{c} \Delta T} 2 \pi r \mathrm{~d} r & = \\
& =-\frac{\mu_{0} I_{\mathrm{c}} \frac{\partial J_{\mathrm{c}}}{\partial \mathrm{T}}}{4 \pi C} \Delta I[\log (1-i)+i]
\end{aligned}
$$

où

$$
i=\frac{I}{I_{\mathrm{c}}}=\frac{I}{\pi R^{2} J_{\mathrm{c}}}
$$

en appelant $i$ le courant réduit.

Suivant le critère de Hancox [5], la pénétration de courant deviendra instable pour le courant

$$
I_{\text {ins }}=i_{\text {ins }} I_{\mathrm{c}}
$$

quand le rapport $\left|\Delta I_{\Delta \mathrm{T}}\right| / \Delta I$ sera égal à 1 . Le courant réduit correspondant à l'amorçage de l'instabilité $i_{\text {ins }}$ peut donc être calculé de l'équation suivante :

$$
\begin{aligned}
\left|\frac{\Delta I_{\Delta T}}{\Delta I}\right| & = \\
& =\frac{\mu_{0} I_{\mathrm{c}} \frac{\partial J_{\mathrm{c}}}{\partial T}}{4 \pi C}\left(-\log \left(1-i_{\text {ins }}\right)-i_{\text {ins }}\right)=1
\end{aligned}
$$

soit

$$
\begin{aligned}
\frac{4 \pi C}{\mu_{0} I_{\mathrm{c}}\left|\frac{\partial J_{\mathrm{c}}}{\partial T}\right|} & = \\
\quad= & -\left[i_{\text {ins }}+\log \left(1-i_{\text {ins }}\right)\right]=\eta\left(i_{\text {ins }}\right) .
\end{aligned}
$$

Expression analogue à celle obtenue par Walters [10]. Comme $i_{\text {ins }}$ peut varier entre 0 et 1 , nous voyons de (10b) que contrairement au cylindre placé dans un champ parallèle pour un fil rond, il existe toujours une valeur de $i_{\text {ins }}$ quels que soient $I_{\mathrm{c}}, \partial J_{\mathrm{c}} / \partial T$ et $C$.

La condition complémentaire qui doit être encore satisfaite pour que l'instabilité initiée soit une instabilité totale, c'est-à-dire, pour que le courant à partir duquel l'instabilité s'amorce, devienne un courant de blocage $I_{\mathrm{b}}$, est que l'énergie dissipée dans le supraconducteur pendant la pénétration du courant du rayon $r_{0}$ jusqu'à l'axe soit suffisante pour échauffer le supraconducteur de telle façon que le courant critique correspondant à cette température obtenue soit inférieur au courant appliqué. Le calcul de cette énergie dissipée pendant la pénétration brusque du courant jusqu'à l'axe du fil, comme le fait Hancox [6], peut être fait en supposant que la température après la pénétration du courant est pratiquement uniforme. Cette supposition qui est en contradiction avec ce que nous avons supposé pour calculer $i_{\text {ins }}$ conduit à des pertes plus faibles que ce qu'elles sont en réalité.

Ce critère cependant nous permettra dans le cas où il est satisfait, d'affirmer que le courant instable $I_{\text {ins }}$ est aussi le courant de blocage $I_{\mathrm{b}}$. Ces pertes $W_{\text {sf }}$ par unité de longueur, calculées à l'aide du vecteur de Poynting, apparaissent comme la différence de son flux, pendant le saut de flux, et de l'accroissement de l'énergie emmagasinée dans le champ magnétique propre :

Soit :

$$
\begin{aligned}
W_{\mathrm{sf}}=2 \pi R & \int_{0}^{\tau} E(R) H_{I}(R) \mathrm{d} t- \\
& -\left[\int_{0}^{R} \frac{\mu_{0} H_{f}^{2} 2 \pi r \mathrm{~d} r}{2}-\int_{r_{0}}^{R} \frac{\mu_{0} H_{f}^{2} 2 \pi r \mathrm{~d} r}{2}\right]
\end{aligned}
$$

d'où

$W_{\mathrm{sf}}=\frac{-\mu_{0} I_{\mathrm{co}}^{2}}{8 \pi}(1-i) \times$

$$
\times[i+(1+i) \log (1-i)] .
$$

Ceci entraîne le critère suivant pour $I_{\mathrm{b}}$ dû à l'instabilité magnétique :

$$
\begin{aligned}
\pi R^{2} \int_{T_{0}}^{T_{C B I_{\mathrm{b}}}} C \mathrm{~d} T & <-\frac{\mu_{0} I_{\mathrm{co}}^{2}}{8 \pi}\left(1-i_{\mathrm{b}}\right) \times \\
& \times\left[i_{\mathrm{b}}+\left(1+i_{\mathrm{b}}\right) \log \left(1-i_{\mathrm{b}}\right)\right]
\end{aligned}
$$

avec

$$
T_{C B I_{\mathrm{b}}}=T_{0}+\left(1-i_{\mathrm{b}}\right)\left(T_{C B}-T_{0}\right) .
$$


On écrit encore (12) sous la forme :

$$
\begin{aligned}
& \frac{8 \int_{T_{0}}^{T_{C B I_{\mathrm{b}}}} C \mathrm{~d} T}{\mu_{0} J_{\mathrm{co}}^{2}} R^{2} \\
& \quad \times\left[i_{\mathrm{b}}+\left(1+i_{\mathrm{b}}\right) \log \left(1-i_{\mathrm{b}}\right)\right]=\xi\left(i_{\mathrm{b}}\right) .
\end{aligned}
$$

Nous avons résolu graphiquement (Fig. 3-3) les équations $(10 b)$ pour $i_{\text {ins }}$ et $(12 a)$ pour $i_{\mathrm{b}}$ à l'aide des expressions $\eta\left(i_{\text {ins }}\right)$ et $\xi\left(i_{\mathrm{b}}\right)$.

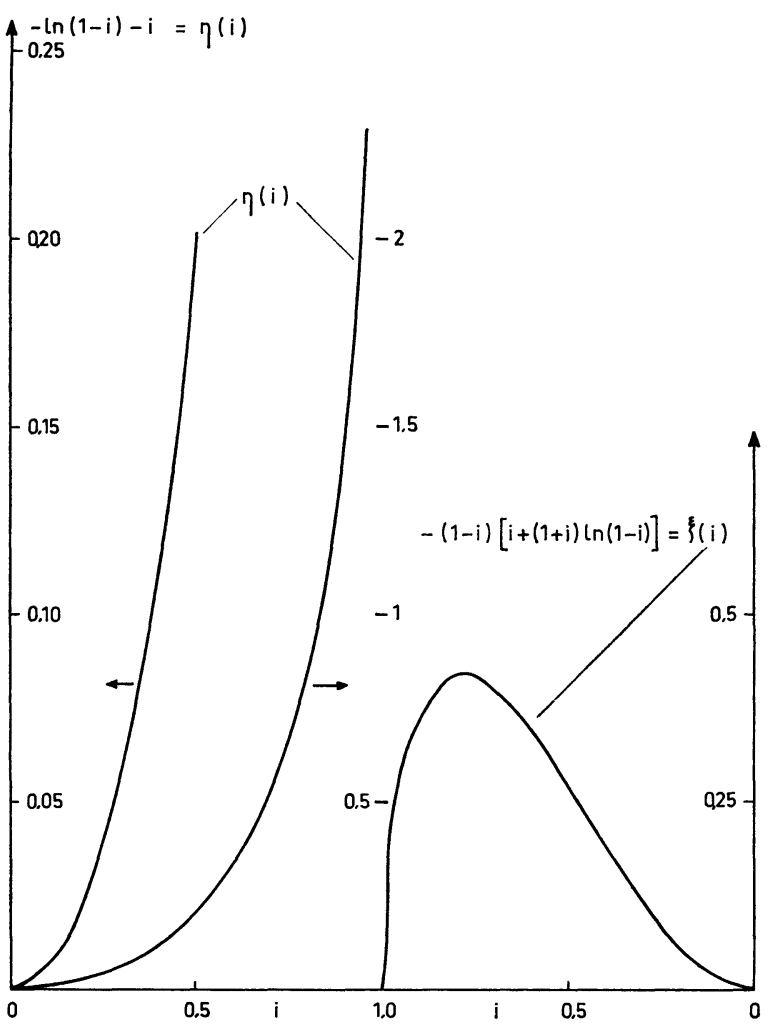

FIG. 3-3. - Résolution graphique de l'équation (12a).

III. 2 RÉSULTATS NUMÉRIQUES. - Les paramètres nécessaires dans les calculs, $R, J_{\mathrm{c}}(B T)$ sont donnés par l'expérience. En ce qui concerne la valeur de la chaleur spécifique $C$ à $4,2^{\circ} \mathrm{K}$, nous trouvons dans la littérature des valeurs allant de $0,05 \mathrm{~mJ} / \mathrm{cm}^{3}{ }^{\circ} \mathrm{K}$ [11] jusqu'à $4,5 \mathrm{~mJ} / \mathrm{cm}^{3} \mathrm{o}$ [12]. La relation indiquée par Hampshire [8] qu'il obtient des références [13] et [14], donne $C$ croissant avec le champ et variant depuis les valeurs 1 à $2 \mathrm{~mJ} / \mathrm{cm}^{3} \mathrm{oK}$ pour des champs de 0 à 6 teslas pour $T=4,2 \mathrm{~K}$. En fonction de $T$ on adopte

$$
\text { pour } T=4,2{ }^{\circ} \mathrm{K} \quad C=\beta T^{3} .
$$

Le comportement thermodynamique des supraconducteurs de $2^{\mathrm{e}}$ espèce entraîne globalement une augmentation de $C$ avec $H$. Cependant, certains résultats expérimentaux [14] et [16] rapportés dans la littérature sous une forme $C / T=f\left[T^{2}\right]$ pour différents champs montrent, par exemple, dans le cas du titane/ molybdène très pur [Barnes et Hake, Cryogenics, avril 1967], une inversion dans la croissance de $C$ avec $H$ pour la température de transition $T_{\mathrm{c}}$ (décroissance avec $H$ ).

Le phénomène de décroissance de $C$ avec $H$ est observable aussi dans le cas du composé Pd-Co à $4,2 \circ \mathrm{K}$ et sous des champs appliqués passant de 0 à 27 kœrsteds, étude donnée en référence [17].

Aussi, sur la figure 2, nous reportons les valeurs $I_{\text {ins }}$ solutions de $(10 b)$ en adoptant $C=4,5-1,5$ et $0,5 \mathrm{~mJ} / \mathrm{cm}^{3}{ }^{\circ} \mathrm{K}$ respectivement pour 1,3 et 5 teslas.

La valeur de $C=4,5 \mathrm{~mJ} / \mathrm{cm}^{3}{ }^{\circ} \mathrm{K}$ pour 0 tesla à $4,2^{\circ} \mathrm{K}$, est celle publiée par Iwasa et Montgomery dans la référence [12] citant l'auteur russe Zbasnik. Pour les autres valeurs à 3 et 5 teslas, elles sont celles qui conformément avec les références citées et en absence de valeurs mesurées concordantes, donnent le meilleur accord entre les mesures et les valeurs $I_{\text {ins }}$ ainsi calculées.

Les courants $I_{\text {ins }}$ satisfont le critère des courants de blocage (équation $12 a$ ) pour $B=1$ tesla, jusqu'à $5,4^{\circ} \mathrm{K}<T<5,8^{\circ} \mathrm{K}$, pour 3 teslas jusqu’à

$$
5^{\circ} \mathrm{K}<T<5,4^{\circ} \mathrm{K},
$$

et pour 5 teslas jusqu'à $4,8^{\circ} \mathrm{K}<T<5^{\circ} \mathrm{K}$. Pour des températures plus élevées, les courants $I_{\text {ins }}$ ne devraient pas être selon ce critère (12a) identiques à des courants de blocage. Or, en effectuant la comparaison par le calcul, nous constatons que même pour des températures légèrement au-dessus de ces valeurs limites, les courants $I_{\mathrm{b}}$ sont pratiquement égaux à des courants $I_{\text {ins. }}$.

Cela est probablement dû au fait que la supposition de température uniforme dans le matériau après le saut de flux conduit à des pertes calculées pendant le saut de flux plus petites que les pertes réelles. D'autre part, il existe aussi les imprécisions sur les mesures qui affectent les calculs par les paramètres $J_{\mathrm{c}}(T, B)$.

Cependant, en ce qui concerne ce premier point (fil rond), l'accord entre les $I_{\text {ins }}$ calculées et les courants de blocage mesurés est très bon, avec les restrictions mentionnées plus haut.

Dans le cas du matériau stabilisé par $80 \mu$ de plomb, on conserve la notion d'adiabaticité du matériau composite global (supraconducteur + matériau plaqué), c'est-à-dire que l'énergie échangée avec le monde extérieur au composite (hélium gaz) est négligeable par rapport à l'énergie développée lors de l'instabilité.

Par ailleurs, on admet que le composite est isotherme.

Dans ces conditions en adoptant comme valeur de chaleur spécifique pour le composite global l'expression :

$$
\underset{\substack{\text { materiau } \\ \text { global }}}{C=} \frac{C_{\text {sup }} S_{\text {sup }}+C_{\text {st }} S_{\mathrm{st}}}{S_{\text {sup }}}
$$

avec $S=$ section du matériau considéré, on détermine pour 1,3 et 5 teslas les valeurs $C=21, \mathrm{~mJ} / \mathrm{cm}^{3}{ }^{\circ} \mathrm{K}$; $18,6 \mathrm{~mJ} / \mathrm{cm}^{3}{ }^{\circ} \mathrm{K} ; 17,6 \mathrm{~mJ} / \mathrm{cm}^{3}{ }^{\circ} \mathrm{K}$ du composite global. 
Dans le cas du placage par $80 \mu$ d'Au en adoptant les mêmes hypothèses, il vient,

$$
C=10,2-7,2-6,2 \mathrm{~mJ} / \mathrm{cm}^{3} \mathrm{oK}
$$

pour 1,2 et 3 T. Nous retrouvons aussi le bon accord avec les résultats expérimentaux (Fig. 4-1).

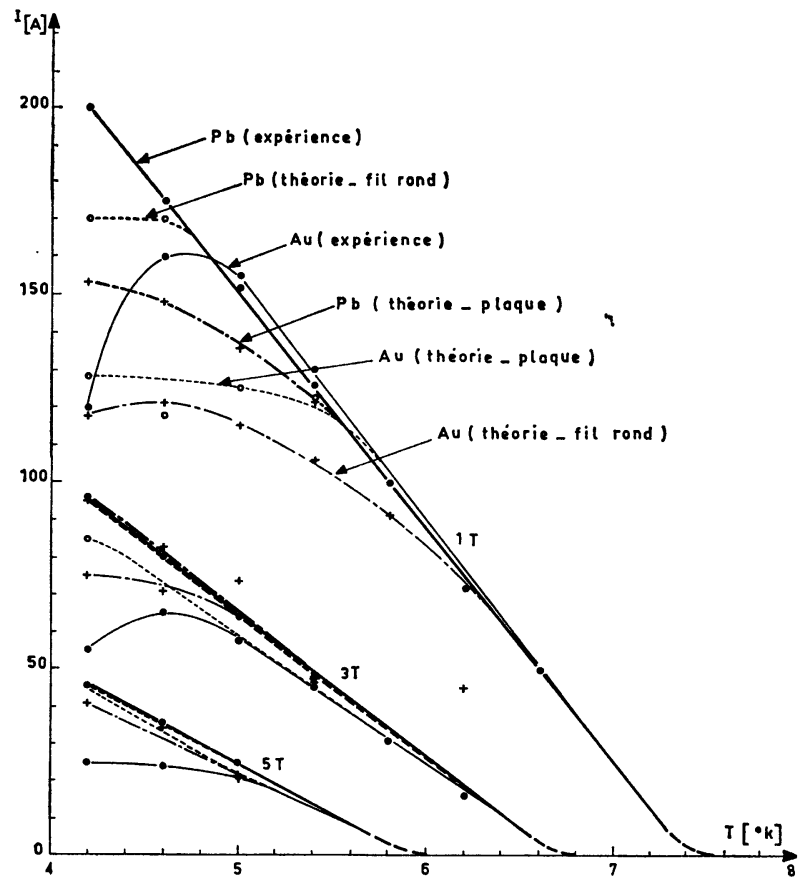

FIG. 4-1. $-I=f[T B] . \mathrm{Nb} 40 \% \mathrm{Ti} . \varnothing=220 \mu$.

Stabilisant $80 \mu\left\{\begin{array}{l}\mathrm{Pb} \\ \mathrm{Au}\end{array}\right.$

Non refroidi dans $H$.

Expérience $\mathrm{Pb}-$

Refroidi dans $H$.
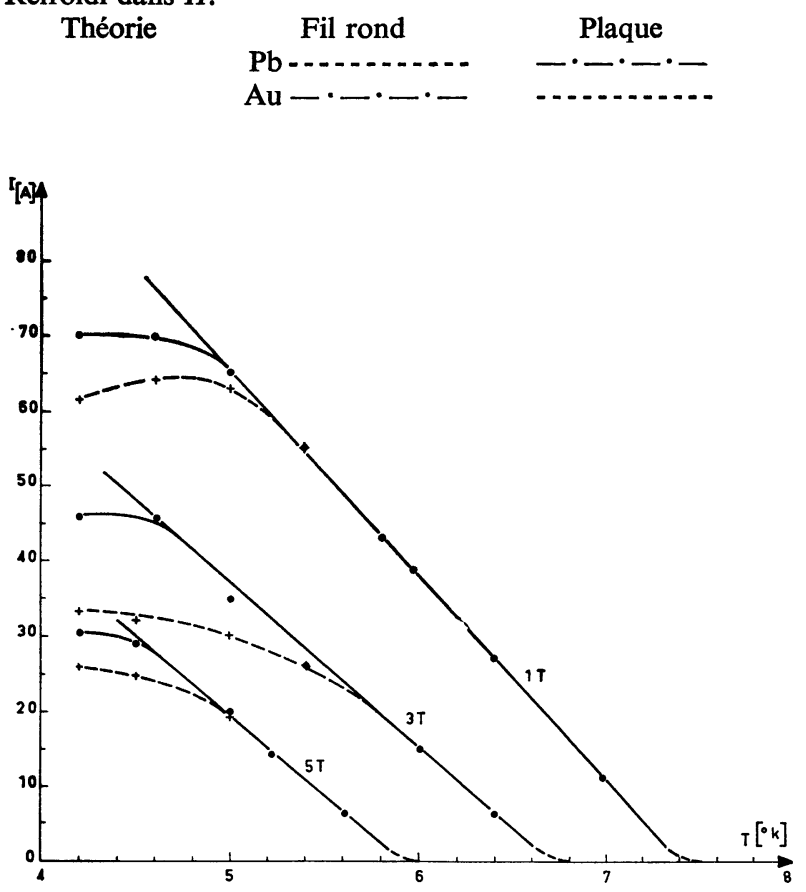

FIG. 4-2. $-I_{\mathrm{b}}=f[T B]$. Echantillon Nb $40 \%$ Ti. $\varnothing 160 \mu$.

Expérience. Non refroidi dans $H$ (Trait Théorie. Refroidi dans $H$, plaque (Trait
IV. Modèle théorique de la plaque (refroidi et nón refroidi dans $\boldsymbol{H}$ ). - On étudie ici (Fig. 5), les conditions d'un saut de flux total dans une plaque dont la surface est identique à celle du fil rond précédemment étudié, toutes choses égales par ailleurs.

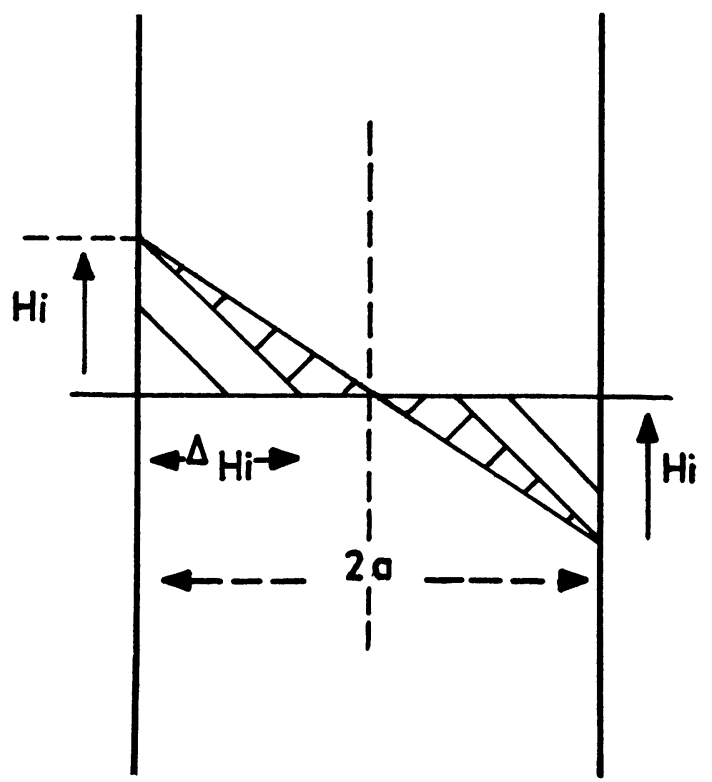

FIG. 5. - Configuration de champ. Echantillon refroidi dans $H$.

IV.1 RefroId DaNs $H$. - En utilisant le vecteur de Poynting, on calcule $W_{\mathrm{fi}}$.

On trouve :

$$
W_{\mathrm{fi}}=\mu_{0} H_{\mathrm{fj}}^{2} \frac{2}{3}\left[a-\Delta \mathrm{f}_{\mathrm{J}}\right] .
$$

Soit :

$$
W_{\mathrm{f}_{\mathrm{t}}} \text { moyen }=\frac{\mu_{0}}{2} H_{\mathrm{f} \mathrm{j}}^{2} \frac{2}{3}\left[1-\frac{\Delta \mathrm{f}_{\mathrm{j}}}{a}\right] .
$$

L'équation (14a) peut s'écrire sous la forme suivante, en faisant apparaître les courants :

$$
W_{\mathrm{fj}} \text { moyen }=\frac{\mu_{0}}{2} \frac{I_{b}^{2}}{16 a^{2}} \frac{2}{3}[1-i] .
$$

En écrivant la condition de saut de flux total pour le courant $I_{\mathrm{b}}$, il vient :

$$
W_{\mathrm{fi}} \text { moyen }=\int_{T_{0}}^{T_{C_{B i}}} C \mathrm{~d} T .
$$

Ce qui entraîne en posant :

$$
\begin{aligned}
I & =1-i=1-\frac{I_{\mathrm{b}}}{I_{\mathrm{c}}} \\
E & =J_{\mathrm{c}}^{2} a^{2} \frac{\mu_{0}}{3} \\
A & =T_{C B}-T_{0}
\end{aligned}
$$$$
I^{4} A^{3} \frac{\beta}{4}+I^{3}\left[T_{0} A^{2} \beta-\frac{E}{A}\right]+
$$$$
+I^{2}\left(6 T_{0}^{2} A \frac{\beta}{4}+\frac{2 E}{A}\right)+I\left(T_{0}^{3} \beta-\frac{E}{A}\right)=0 \text {. }
$$ 
Cette équation paramétrique du $4^{\mathrm{e}}$ degré en

$$
I=1-\frac{I_{\mathrm{b}}}{I_{0}}
$$

fournira des solutions $I_{\mathrm{b}}$ en fonction de la température $T_{0}$ du milieu réfrigérant. Les solutions réelles convenant au problème ne peuvent exister que pour des valeurs bien précises des coefficients. L'existence entre 0 et 1 des solutions entraîne les conditions suivantes sur le terme $T_{0}^{3} \beta-E / A$ à savoir

$$
\beta T_{0}^{3}-\frac{J_{c}^{2} a^{2} \mu_{0}}{3 T_{C B}-T_{0}}<0 .
$$

Cette condition d'existence de racines vérifiant le problème physique peut être présentée alors comme un critère de stabilité. On constate, puisqu'il s'écrit

$$
1<\mu_{0} J_{\mathrm{c}}^{2} a^{2} / 3 C\left[T_{C B}-T_{0}\right],
$$

qu'il est légèrement différent, du moins dans son formalisme, au critère de stabilité de Hancox [5], [6]. Nous étudierons ultérieurement les conséquences de ce critère.

On se borne ici à reporter sur la figure 2 les solutions obtenues par résolution numérique de l'équation (16) à l'aide du programme NOO 6 utilisant la méthode de Baristow et cela avec la chaleur spécifique déjà adoptée dans la configuration cylindrique. Dans cette hypothèse, $C$ décroissant avec $H$, l'accord est très bon entre ces résultats obtenus de $I_{b}$ calculés sur la plaque et ceux obtenus sur le fil rond. Tous deux confirment les mesures réalisées. Le modèle de la plaque étant ainsi justifié, il est possible de façon simple à l'aide du vecteur de Poynting, de reprendre le calcul des pertes lorsqu'il existe dans le matériau des courants diamagnétiques.

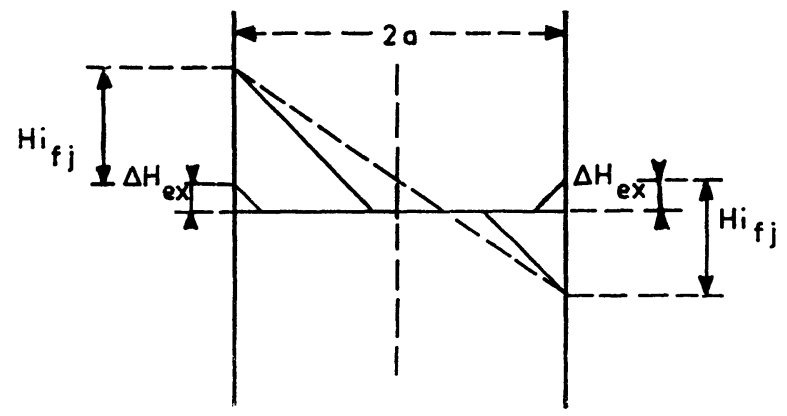

FIG. 6-1. - $1^{\circ}$ Configuration de champ. Echantillon avec courants diamagnétiques non refroidi dans $H$.
IV.2 Partiellement Refroid dans $H_{\text {. }}$ - Nous étudions les deux cas des figures 6-1 et 6-2.

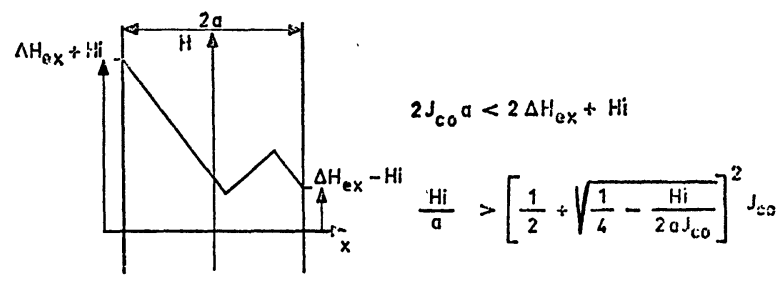

FIG. 6-2. - 20 Configuration de champ. Echantillon avec courants diamagnétiques non refroidi dans $H$.

$$
\begin{aligned}
2 J_{\mathrm{co}} a & <2 \Delta H_{\mathrm{ex}}+H^{\mathrm{i}} \\
\frac{H_{\mathrm{i}}}{a}> & >\frac{1}{2}+\sqrt{\left.\frac{1}{4}-\frac{H_{\mathrm{i}}}{2 a J_{\mathrm{co}}}\right]} J_{\mathrm{co}} .
\end{aligned}
$$

En ce qui concerne le profil de champ de la figure 6-1, c'est-à-dire $H_{\text {ex }}$ sur lequel on superpose à droite et à gauche le champ $H_{\mathrm{i}}$ (propre dû au courant), nous écrirons alors :

$$
\begin{aligned}
\int S \mathrm{~d} t= & \frac{\mu_{0}}{2}\left[\frac{1}{J_{\mathrm{cf}}}-\frac{1}{J_{\mathrm{ci}}}\right] \times \\
& \times\left[\left[\Delta H_{\mathrm{ex}}+H_{\mathrm{i}}\right]^{3}+\left[H_{\mathrm{i}}-\Delta H_{\mathrm{ex}}\right]^{3}\right] \\
W_{\text {moy }}= & \frac{\mu_{0}}{2} \cdot\left[\frac{1}{3 J_{\mathrm{cf}}}-\frac{1}{3 J_{\mathrm{ci}}}\right] \times \\
& \times\left[\left[\Delta H_{\mathrm{ex}}+H_{\mathrm{i}}\right]^{3}+\left[H_{\mathrm{i}}-\Delta H_{\mathrm{ex}}\right]^{3}\right] .
\end{aligned}
$$

L'expression des pertes moyennes dissipées pendant le saut de flux est :

$$
\begin{aligned}
W_{\mathrm{fi}}=\frac{\mu_{0}}{4 a}\left[H_{\mathrm{i}}\right. & \left.+\Delta H_{\mathrm{ex}}\right]^{3}+ \\
& +\left[H_{\mathrm{i}}-\Delta H_{\mathrm{ex}}\right]^{3} \frac{2}{3}\left[\frac{1}{J_{\mathrm{cf}}}-\frac{1}{J_{\mathrm{ci}}}\right] .
\end{aligned}
$$

Pour le profil (6-2) en tenant compte de l'expulsion du flux puisque par hypothèse on a supposé une distribution en température homogène dans tout le supraconducteur lors du saut de flux, il vient comme expression des pertes moyennes en posant :

$$
\begin{aligned}
& 2 J_{\mathrm{co}} a<2 \Delta H_{\mathrm{ex}}+H_{\mathrm{i}} \quad \mid W_{\mathrm{fi}}=\frac{\mu_{0} H_{\mathrm{i}}^{2}}{2}\left\{\left(\frac{\Delta H_{\mathrm{ex}}}{H_{\mathrm{i}}}\right)^{2}\left[1+\sqrt{\frac{a J_{\mathrm{co}}}{4 H_{\mathrm{i}}}}\left(1-2 \sqrt{\frac{a J_{\mathrm{co}}}{H_{\mathrm{i}}}}-\frac{a J_{\mathrm{co}}}{H_{\mathrm{i}}}\right)\right]+\right. \\
& +\frac{\Delta H_{\mathrm{ex}}}{H_{\mathrm{i}}}\left[\left\{1-\frac{a J_{\mathrm{co}}}{H_{\mathrm{i}}}\left(1+\sqrt{\frac{a J_{\mathrm{co}}}{4 H_{\mathrm{i}}}}\right)\right\}^{2}-\frac{a J_{\mathrm{co}}}{4 H_{\mathrm{i}}}\right]+\left(\frac{a J_{\mathrm{co}}}{H_{\mathrm{i}}}\right)^{2} \\
& \frac{H_{\mathrm{i}}}{a}>\left[\frac{1}{2}+\sqrt{\frac{1}{4}-\frac{H_{\mathrm{i}}}{2 a J_{\mathrm{co}}}}\right]^{2} J_{\mathrm{co}}\left[\left[\frac{1}{3}+\left(1+\sqrt{\frac{a J_{\mathrm{co}}}{4 H_{\mathrm{i}}}}\right)^{2}-\frac{a J_{\mathrm{co}}}{3 H_{\mathrm{i}}}\left(1+\sqrt{\frac{a J_{\mathrm{co}}}{4 H_{\mathrm{i}}}}\right)^{3}\right]+\frac{1}{4}\right. \\
& \left.-\frac{a J_{\mathrm{co}}}{4 H_{\mathrm{i}}}\left(5+\frac{11}{6} \sqrt{\frac{a J_{\mathrm{co}}}{H_{\mathrm{i}}}}\right)+\left(1+\frac{a J_{\mathrm{co}}}{2 H_{\mathrm{i}}}\right) \log \sqrt{\frac{a J_{\mathrm{co}}}{H_{\mathrm{i}}}}\right) \text {. }
\end{aligned}
$$


L'ensemble de ces calculs montre que pour certaines valeurs de $\Delta H_{\mathrm{ex}}$ croissantes, il doit exister des instabilités entraînant périodiquement des courants de blocage $I_{\mathrm{b}}$ très proches de zéro. Des essais en cours semblent confirmer cette hypothèse et permettent donc de mesurer $\Delta H_{\mathrm{ex}}$ entraînant une dégradation importante.

En ce qui concerne les calculs effectués à partir de l'équation (16) sur le matériau plaqué au $\mathrm{Pb}$ et l' $\mathrm{Au}$, on trouvera les résultats reportés sur la figure 4-1; ils vérifient bien les résultats expérimentaux. Il en est de même quant à l'influence de la section du supraconducteur existant dans l'équation (16) sur le coefficient $E / A$. En effet, les courants de blocage calculés comme étant les solutions de (16) dans le cas où la section du matériau est de $160 \mu$, vérifient à $20 \%$ les expériences qui, on le souligne dans ce cas, étaient faites sans refroidir au préalable le matériau dans le champ (Fig. 4-2).

L'accord entre les solutions calculées par l'équation (16) et les mesures est assez bien réalisé pour confirmer l'adoption dans le cas du matériau stabilisé du modèle de la plaque.

V. Conclusion. - Nos mesures permettent de mettre en évidence le rôle essentiel des instabilités magnétiques et montrent qu'il est donc possible de détecter directement par une mesure de courant sur des échantillons courts dans les conditions expérimentales décrites, les phénomènes de saut de flux. Les modèles proposés (fil rond et plaque) confirment et expliquent 1'allure générale des courbes expérimentales qui diffèrent sensiblement dans certaines plages de tempé- rature et de champs, des droites théoriques $J_{\mathrm{c}}(T)$. En ce qui concerne le modèle de la plaque, il permettra de calculer simplement l'influence sur les courants de transport des instabilités entraînées par la variation du champ de polarisation appliqué, ou du champ propre, lorsque le matériau n'est pas refroidi totalement dans le champ. En effet, comme l'ont montré nos mesures effectuées sur échantillons $\mathrm{A}$ et $\mathrm{B}$, le training qui disparaît totalement lorsque le matériau est refroidi au préalable dans le champ, peut être relié à l'existence des courants diamagnétiques.

D'une manière générale, l'indétermination de la fonction $C=f[H]$ ne paraît pas devoir remettre en cause dans les modèles, le rôle essentiel de la chaleur spécifique ainsi que la taille du matériau sur les performances du supraconducteur.

La variation de $C$ dépend très fortement du pourcentage des matériaux simples composant le supraconducteur [15]. Il est donc difficile d'adopter des valeurs mesurées existant dans la littérature, mais dont les pourcentages relatifs de $\mathrm{Nb}$ et $\mathrm{Ti}$ ne sont pas identiques au supraconducteur étudié ici.

Il semble bien aussi [16], qu'aux alentours de $T_{\mathrm{c}}$ ( $T_{\mathrm{c}}$ critique) variant en fonction de $H$ la chaleur spécifique $C$ peut décroître pour des champs appliqués croissants. D'autre part, la littérature fournit des valeurs de $C$ pour un matériau qui peut varier considérablement.

Il sera donc nécessaire de préciser ultérieurement en fonction de $H$ (pour le matériau étudié) les valeurs de $C$, et de tenir compte de la diffusivité thermique du matériau, paramètres susceptibles de modifier les valeurs de courant $I_{\mathrm{b}}$ calculées ici.

\section{Bibliographie}

[1] Kim (Y. B.), Hemptead (C. F.) and Strnad (A. R.), Phys. Rev. Letters, 1962, 9, 306.

[2] Hancox (R.), Phys. Letters, 1965, 16, 208.

[3] Wipf (J. L.), Phys. Rev., 1967, 161, 404.

[4] Swartz (P. S.) et Bean (C. P.). - J. Appl. Phys., 1968, 39, 4991.

[5] Hancox (R.), Appl. Phys. Letters, 1965, 7, 138.

[6] Hancox (R.), ( Moscow Proceeding of 10th International Conference on how temperature. ) Physics, 1966.

[7] Smith (P. F.), Wilson (M. N.), Walters (C. R.) and LEWIN (J. D.), Rutherford Laboratory RPP/A 73.

[8] Hampshire (R.), Sutton (J.) et Taylor (M. T.), (Conférence sur les basses températures et l'énergie électrique. ) Londres 24/26 mars 1969.
[9] Yamafuji (K.), Takeo, Chikaba (J.), Yano, J. Phys. Soc. Japan, 1969, 26, 315.

[10] WALTERS (C. R.), Rutherford Laboratory RPP/A 73.

[11] Chikaba (J.), Cryogenics 10, 306 (August 1970).

[12] IWASA (Y. I.), WegGelC, Montgomery (D. B.), Weggel (R.) and Hale (J. R.), J. Appl. Phys., 1969, 40.

[13] Sukharewsky (B. Y.), Alapina (A. V.), Dushechkin (A.), Th. Elesp teor Fiz, 1968, 54, 1675.

[14] Ferreira (J.), Burgemeister (E. A.), Dokoupil (Z.), Phys. Letters, 1967, 25A, 354.

[15] Morin (F. J.), Maita (J. P.), Phys. Rev., 1963, 129.

[16] Hake (R. R.), J. Appl. Physics, 1969, 40.

[17] Boerstoel (B. M.), Van BaArle (C.), J. Appl. Physics, 1970, 41. 